\title{
Comportamento alimentar e alterações sensoriais em pacientes em quimioterapia
}

\author{
Eating behavior and sensory changes in patients undergoing chemotherapy
}

DOI: $10.37111 /$ braspeni.2020353009

Otávio Augusto Moura Fernandes'

Larissa Casari ${ }^{\top}$

Vera Lúcia Ferreira da Silva?

Laura Moreira Goularte

Shirley Sousa de Oliveira ${ }^{2}$

Karina Sanches Machado d'Almeida ${ }^{3}$

Anne y Castro Marques ${ }^{4}$

\section{Unitermos:}

Antineoplásicos. Neoplasias. Distúrbios do paladar. Transtornos do olfato. Comportamento alimentar.

\section{Keywords:}

Antineoplastic agents. Neoplasms. Taste disorders. Olfaction disorders. Feeding Behavior.

\section{Endereço para correspondência:}

Otávio Augusto Moura Fernandes

Rua Gomes Carneiro, 1 - Bairro Centro - Pelotas, RS,

Brasil - CEP: $96075-630$

E-mail: otavioaugustomofe@gmail.com

\section{Submissão}

7 de março de 2020

\section{Aceito para publicação}

3 de setembro de 2020

\begin{abstract}
RESUMO
Introdução: A quimioterapia é um tipo de tratamento amplamente utilizado contra o câncer, mas que pode resultar em alterações sensoriais e em aversão por alimentos habitualmente consumidos antes do tratamento, repercutindo negativamente no estado nutricional do paciente. $\mathrm{O}$ objetivo deste trabalho foi avaliar o comportamento alimentar e as possíveis alterações de paladar e olfato em pacientes em quimioterapia no Hospital Escola da Universidade Federal de Pelotas (UFPel). Método: Estudo transversal realizado no primeiro semestre de 2019, com pacientes acima de 18 anos, a partir do segundo ciclo de quimioterapia. A coleta de dados foi realizada por meio de um questionário, contendo informações sociodemográficas e relacionadas ao tratamento, presença de sinais e sintomas e aversão alimentar adquirida. Os dados foram analisados por meio de análise descritiva, e para comparação das variáveis categóricas entre os momentos antes e após o tratamento quimioterápico foi utilizado o teste qui-quadrado de Pearson, com $p<$ 0,001. Resultados: Participaram do estudo 101 pacientes oncológicos, sendo a maioria do sexo feminino $(58,4 \%)$. Os sítios iniciais de tumor de maior prevalência foram trato gastrointestinal e mama. Os alimentos que apresentaram estatisticamente alteração do comportamento alimentar $(p<0,001)$ foram: feiião, vegetais folhosos, legumes, leite e derivados, pães/bolos, sopas, refrigerantes/doces. Quanto à aversão alimentar apreendida, os alimentos que mais se destacaram foram refrigerantes/doces e embutidos. Os efeitos adversos oriundos do tratamento mais citados foram: alteração ou redução do sabor dos alimentos, xerostomia e enjoo oriundo do aroma dos alimentos. Conclusões: A quimioterapia afeta a relação do paciente com a comida, provocando uma importante alteração no comportamento alimentar.
\end{abstract}

\section{ABSTRACT}

Introduction: Chemotherapy is a type of treatment widely used against cancer, but it can result in sensory changes and aversion to foods usually consumed before treatment, negatively impacting the patient's nutritional status. The objective of this study was to assess the feeding behavior and the possible changes of taste and smell in patients undergoing chemotherapy at the Hospital School of Universidade Federal de Pelotas (UFPel). Methods: A cross-sectional study was conducted in the first half of 2019, with patients above 18 years, in the second cycle of chemotherapy. The data collection was performed from a self-administered questionnaire containing sociodemographic and treatment-related information, presence of signs and symptoms, and acquired food aversion. Data were analyzed using descriptive analysis, and to compare categorical variables between the moments before and after chemotherapy, Pearson's chi-square test was used, with $p<0.001$. Results: A total of 101 cancer patients participated in the study, most of them female (58.4\%). The initial tumor sites of higher prevalence were in the gastrointestinal tract and in the breast. Foods that showed statistically altered dietary pattern $(p<0.001)$ were: beans, leafy vegetables, vegetables, milk and dairy products, bread/cakes, soups, soft drinks/sweet drinks. Regarding the food aversion seized, the food that stood out the most was soft drinks/sweets and processed meat. The most frequently cited adverse effects of the treatment were: alteration or reduction in the taste of food, xerostomia and nausea caused by the food's aroma. Conclusion: Chemotherapy affects the patient's relationship to food, causing an important change in eating behavior.

1. Graduados em Nutrição pela Universidade Federal de Pelotas, Pelotas, RS, Brasil.

2. Bacharel pela Universidade Federal de Santa Catarina, Nutricionista em Hospital Universitário Polydoro Ernani de São Thiago, Florianópolis, SC, Brasil.

3. Doutora em Ciências da Saúde: Cardiologia e Ciências Cardiovasculares/Universidade Federal do Rio Grande do Sul, Professora do Curso de Nutrição/ Universidade Federal do Pampa, Itaqui, RS, Brasil.

4. Doutora em Alimentos e Nutrição/ Universidade Estadual de Campinas, Professora do Curso de Nutrição/ Universidade Federal de Pelotas, Pelotas, RS, Brasil. 


\section{INTRODUÇÃO}

Câncer, tumor maligno e neoplasia maligna são termos utilizados para nomear doenças relacionadas ao rápido crescimento de células anormais, além de seus limites habituais, que podem invadir outros tecidos'. Muitos fatores influenciam o desenvolvimento do câncer e podem ser externos, como meio ambiente, hábitos ou costumes próprios de um ambiente social e cultural, ou internos, resultantes de eventos que geram mutações sucessivas no material genético das células, processo que pode ocorrer ao longo de décadas, em múltiplos estágios².

De acordo com a Organização Pan-Americana da Saúde ${ }^{1}$, o câncer é uma das principais causas de morte no mundo, sendo responsável por cerca de 9,6 milhões de mortes em 2018. Estima-se, para o Brasil, biênio 2018/2019, a ocorrência de 600 mil casos novos de câncer por ano. Excetuando-se o câncer de pele não melanoma (cerca de 170 mil casos novos), ocorrerão 420 mil casos novos de câncer. Os cânceres de próstata $(68 \mathrm{mil})$ em homens e mama (60 mil) em mulheres serão os mais frequentes ${ }^{3}$. Uma projeção da International Agency for Research Cancer (IARC) indica que, no Brasil, em 2030, haverá 733.340 novos casos da doença, excetuando-se o de pele não melanoma, representando um aumento de aproximadamente $54 \%$ em comparação com $2015^{4}$.

A quimioterapia é um tipo de tratamento endovenoso, intravesical e/ou oral em que se utilizam medicamentos para combater o câncer. Estes fármacos alcançam todos os tecidos do organismo, destruindo as células que estão formando o tumor e impedindo que se espalhem ${ }^{5}$. Uma vez que as drogas utilizadas não são seletivas para atacar apenas células malignas, a destruição de células saudáveis pode repercutir em uma série de efeitos adversos, tais como: náuseas, vômitos, anormalidades no paladar, mucosite, estomatite, diarreia e constipação. Tais efeitos adversos podem proporcionar redução da ingestão alimentar e, consequentemente, depleção do estado nutricional, elevando, assim, os índices de morbimortalidade ${ }^{6-8}$.

Nesse contexto, a relação do indivíduo com o alimento pode mudar durante a quimioterapia; preparações saborosas antes do tratamento podem se tornar desagradáveis ou provocar mal-estar, e uma alteração hedônica pode ocorrer, o que significa que, embora o alimento tenha o mesmo gosto, o sabor não é mais considerado como agradável ${ }^{9}$. De acordo com Verde et al. ${ }^{10}$, o desenvolvimento de aversões alimentares resulta da associação entre o mal-estar promovido pelo tratamento e o alimento simultaneamente consumido.

Além da aversão alimentar adquirida durante o tratamento quimioterápico, o paciente pode também apresentar redução de paladar e olfato. Estudos apontam que mais de 50\% dos pacientes oncológicos referiram alterações de paladar, e 35\% a $87 \%$ alterações de olfato, em decorrência do tratamento. Obviamente, a perda de sabor e aroma agradável da comida provocarão desinteresse destes indivíduos pela alimentação e modificação dos hábitos alimentares ${ }^{11}$.

Levando-se em consideração que o câncer é uma das maiores causas de morte no mundo, e tendo a quimioterapia como uma das formas mais utilizadas de tratamento, a qual possui várias reações adversas, o presente estudo terá como objetivo avaliar o comportamento alimentar e as possíveis alterações de paladar e olfato em pacientes em quimioterapia em um hospital público no sul do país.

\section{MÉTODO}

Tratou-se de um estudo transversal, realizado na cidade Pelotas, Rio Grande do Sul, Brasil, no primeiro semestre de 2019. A amostra foi composta por homens e mulheres com mais de 18 anos, a partir do segundo ciclo de quimioterapia e que aceitaram participar da pesquisa mediante a assinatura do Termo de Consentimento Livre e Esclarecido (TCLE). Foram excluídos gestantes e pacientes incapazes de responder ao questionário. Todos os pacientes que estavam na sala de espera aguardando atendimento foram convidados a participar.

Os dados foram obtidos por acadêmico do curso de Nutrição da Universidade Federal de Pelotas (UFPel), no setor de Quimioterapia do Hospital Escola. As informações sociodemográficas (sexo, idade, cor da pele, estado civil e profissão) foram coletadas, e a classe social foi definida mediante aplicação do questionário da Associação Brasileira de Empresas de Pesquisa ${ }^{12}$. Também foi questionado o tipo de câncer, a presença ou não de metástase e a existência de outras doenças crônicas.

Para identificar a prevalência de alterações de paladar e olfato relacionada ao tratamento quimioterápico, foi utilizada parte da Avaliação Subjetiva Global Produzida Pelo Paciente (ASG/PPP) ${ }^{13}$. Já para identificar a ocorrência de aversão alimentar adquirida, assim como os alimentos com maior aversão, os entrevistados responderam à seguinte pergunta: "Antes de realizar a primeira sessão de quimioterapia, o senhor (a) consumia esses alimentos? Indique sim ou não". Os alimentos investigados foram escolhidos de acordo com o estudo de Ferreira et al. ${ }^{14}$ : arroz, feijão, vegetais folhosos, embutidos, café, carnes, pães/bolos, leite e derivados, frutas, legumes, ovos, massas, refrigerantes/doce e sopas.

Ainda em relação aos sintomas avaliados, destaca-se que foram utilizados os seguintes conceitos ${ }^{15}$ : anorexia $=$ diminuição ou perda de apetite acompanhada por uma aversão à comida e incapacidade para comer; mucosite = inflamação da mucosa do trato gastrointestinal com ulceração; xerostomia = diminuição do fluxo salivar (boca seca); disgeusia = alteração do paladar; e disosmia = perda ou capacidade prejudicada de sentir cheiro.

Os dados coletados foram digitados no programa Microsoft Excel e revisados. A análise dos dados foi 
realizada por meio de análise descritiva, e foram apresentados como porcentagem ou média \pm desvio padrão. Para comparação das variáveis categóricas entre os momentos antes e após o tratamento quimioterápico foi utilizado o teste qui-quadrado de Pearson, com $p<0,001$, no programa estatístico Statistical Package for the Social Sciences (SPSS) versão 18.0.

Esse trabalho fez parte de um projeto maior, intitulado "Hábitos alimentares e qualidade de vida de pacientes em quimioterapia", aprovado pelo Comitê de Ética em Pesquisa da Faculdade de Medicina da UFPel, sob o parecer número 2.927.703.

\section{RESULTADOS}

De 116 pacientes que foram convidados a participar desta pesquisa, 15 foram excluídos por recusarem ou por não estarem em condições para responder. A amostra estudada, portanto, constituiu-se de 101 pacientes oncológicos, sendo a maioria do sexo feminino, entre 24 e 91 anos. Os demais dados podem ser observados na Tabela 1.

Tabela 1 - Dados sociodemográficos de pacientes em tratamento quimioterápico de um hospital público em Pelotas, RS, 2019. n=101.

\begin{tabular}{|c|c|c|}
\hline Variável & $\mathbf{N}$ & $\%$ ou média (DP) \\
\hline \multicolumn{3}{|l|}{ Sexo } \\
\hline Feminino & 59 & 58,4 \\
\hline Masculino & 42 & 41,6 \\
\hline \multicolumn{3}{|l|}{ Idade } \\
\hline Idade (anos)* & 58,6 & $(12,2)$ \\
\hline \multicolumn{3}{|l|}{ Cor da pele } \\
\hline Brancos & 78 & 77,2 \\
\hline Não brancos & 23 & 22,8 \\
\hline \multicolumn{3}{|l|}{ Estado civil $(n=100)$} \\
\hline Solteiro & 23 & 23,0 \\
\hline Casado & 53 & 53,0 \\
\hline Divorciado & 12 & 12,0 \\
\hline Viúvo & 12 & 12,0 \\
\hline \multicolumn{3}{|l|}{ Classe social } \\
\hline Classe A & 4 & 4,0 \\
\hline Classes B1 e B2 & 24 & 23,8 \\
\hline Classes C1 e C2 & 51 & 50,4 \\
\hline Classe D/E & 22 & 21,8 \\
\hline \multicolumn{3}{|l|}{ Profissão (n=100) } \\
\hline Aposentado(a) & 32 & 32,0 \\
\hline Dona de casa & 12 & 12,0 \\
\hline Doméstica/cozinheiro(a) & 12 & 12,0 \\
\hline Agricultor/pecuarista & 7 & 7,0 \\
\hline Professor(a) & 5 & 5,0 \\
\hline Área da saúde & 3 & 3,0 \\
\hline Outros & 29 & 29,0 \\
\hline
\end{tabular}

* Idade mínima: 24 anos; idade máxima: 91 anos.
Na Tabela 2, é apresentada a caracterização do estado de saúde dos pacientes avaliados. Foi possível observar que os tumores iniciais com maiores prevalências foram os do trato gastrointestinal e de mama, e que a maioria dos sujeitos não apresentava metástase ou outras doenças crônicas.

Tabela 2 - Dados de saúde de pacientes em tratamento quimioterápico de um hospital público em Pelotas, RS, 2019. n=101.

\begin{tabular}{|c|c|c|}
\hline Variável & $\mathrm{N}$ & $\%$ \\
\hline \multicolumn{3}{|l|}{ Local do tumor inicial } \\
\hline Trato gastrointestinal & 35 & 34,6 \\
\hline Intestino & 22 & 21,8 \\
\hline Esôfago/estômago & 7 & 6,9 \\
\hline Fígado/pâncreas & 6 & 5,9 \\
\hline \multicolumn{3}{|l|}{ Outros locais } \\
\hline Mama & 28 & 27,8 \\
\hline Pulmão & 10 & 9,9 \\
\hline Ginecológico & 10 & 9,9 \\
\hline Próstata/testículo & 7 & 6,9 \\
\hline Pele & 3 & 3,0 \\
\hline Cabeça/pescoço & 2 & 2,0 \\
\hline Outros & 6 & 5,9 \\
\hline \multicolumn{3}{|l|}{ Diagnóstico de metástase } \\
\hline Não & 69 & 68,3 \\
\hline $\operatorname{Sim}$ & 32 & 31,7 \\
\hline \multicolumn{3}{|l|}{ Doenças associadas } \\
\hline Hipertensão & 25 & 24,8 \\
\hline Diabetes & 8 & 7,9 \\
\hline Hipertensão e diabetes & 4 & 4,0 \\
\hline Outras & 4 & 4,0 \\
\hline Sem comorbidades & 60 & 59,4 \\
\hline
\end{tabular}

Os dados referentes ao comportamento alimentar dos pacientes oncológicos, antes e depois do início do tratamento quimioterápico, são apresentados na Tabela 3. Os alimentos que apresentaram estatisticamente alteração de consumo $(p<0,001)$ foram: feijão, vegetais folhosos, legumes, leite e derivados, pães/bolos, sopas e refrigerantes/doces. Quanto à aversão alimentar apreendida, os alimentos que mais se destacaram foram os refrigerantes/doces e os embutidos.

$\mathrm{Na}$ Tabela 4, são apresentados os dados referentes à ingestão alimentar e às alterações de paladar e olfato, segundo a ASG-PPP. Quanto à quantidade, a maioria dos pacientes informou não ter tido mudanças na ingestão alimentar no último mês. Em relação aos efeitos adversos oriundos do tratamento, os mais citados foram: alteração ou redução do sabor dos alimentos, xerostomia e enjoo oriundo do aroma dos alimentos. 
Tabela 3 - Aversão alimentar apreendida entre pacientes em tratamento quimioterápico de um hospital público em Pelotas, RS, 2019.

\begin{tabular}{|c|c|c|c|c|c|}
\hline Alimento & $\mathrm{n}$ & $\begin{array}{c}\text { Aceitação antes da } \\
\text { quimioterapia } \\
\text { n (\%) }\end{array}$ & $\begin{array}{c}\text { Aceitação } \\
\text { pós-quimioterapia } \\
\mathrm{n}(\%)\end{array}$ & Valor $\mathrm{p}^{*}$ & $\begin{array}{c}\text { Aversão alimentar } \\
\text { apreendida** } \\
\text { n (\%) }\end{array}$ \\
\hline Arroz & 97 & $94(96,9 \%)$ & $88(90,7 \%)$ & 0,001 & $7(7,2 \%)$ \\
\hline Feijão & 98 & $95(96,9 \%)$ & $90(91,8 \%)$ & 0,000 & $6(6,1 \%)$ \\
\hline Frutas & 97 & $94(96,9 \%)$ & $92(94,8 \%)$ & 0,025 & $4(4,1 \%)$ \\
\hline Vegetais folhosos & 99 & $87(87,9 \%)$ & $82(82,8 \%)$ & 0,000 & $10(10,1 \%)$ \\
\hline Legumes & 100 & $95(95,0 \%)$ & $88(88,0 \%)$ & 0,000 & $8(8,0 \%)$ \\
\hline Embutidos & 100 & $85(85,0 \%)$ & $52(52,0 \%)$ & 0,033 & 37 (37\%) \\
\hline Carnes & 96 & $90(93,8 \%)$ & $80(83,3 \%)$ & 0,001 & $12(12,5 \%)$ \\
\hline Ovos & 98 & $94(95,9 \%)$ & $91(92,8 \%)$ & 0,001 & $5(5,1 \%)$ \\
\hline Leite/derivados & 99 & $80(80,8 \%)$ & $76(76,8 \%)$ & 0,000 & $10(10,1 \%)$ \\
\hline Pães/bolos & 95 & $90(94,7 \%)$ & $79(83,2 \%)$ & 0,000 & $12(12,6 \%)$ \\
\hline Massas & 99 & $96(97,0 \%)$ & $84(84,8 \%)$ & 0,011 & $13(13,1 \%)$ \\
\hline Sopas & 98 & $93(94,9 \%)$ & $91(92,9 \%)$ & 0,000 & $3(3,1 \%)$ \\
\hline Café & 96 & $90(93,7 \%)$ & $80(83,3 \%)$ & 0,001 & $12(12,5 \%)$ \\
\hline Refrigerante/doces & 100 & $78(78,0 \%)$ & $42(42,0 \%)$ & 0,000 & $38(38 \%)$ \\
\hline
\end{tabular}

* Considerou-se $p<0,001$, segundo o teste qui-quadrado de Pearson. ${ }^{*}$ Considerou-se com aversão alimentar apreendida o sujeito que consumia 0 alimento antes da quimioterapia e passou a rejeitá-lo após o início do tratamento.

Tabela 4 - Ingestão alimentar e alterações de paladar e olfato entre pacientes em tratamento quimioterápico de um hospital público em Pelotas, RS, 2019.

\begin{tabular}{lll}
\hline Variável & $\mathbf{n}$ & $\%$ \\
\hline Ingestão alimentar no último mês & 99 & \\
Sem mudanças & 42 & 42,4 \\
Mais que o normal & 25 & 25,3 \\
Menos que o normal & 32 & 32,3 \\
\hline Sintomas & 100 & \\
Anorexia & 42 & 42,0 \\
Mucosite & 26 & 26,0 \\
Xerostomia & 54 & 54,0 \\
Disgeusia & 66 & 66,0 \\
Disosmia & 43 & 43,0 \\
\hline
\end{tabular}

Os valores ultrapassam $100 \%$, pois os pacientes podem ter relatado mais de um efeito colateral.

\section{DISCUSSÃO}

Este estudo verificou o comportamento alimentar e as alterações de paladar e olfato em pacientes oncológicos durante o tratamento quimioterápico, a partir do segundo ciclo de quimioterapia. Verificou-se a maior prevalência de pacientes do sexo feminino, com média de idade de 58,6 anos, casado, da classe social média-baixa e aposentado, dados semelhantes aos encontrados em outros estudos ${ }^{16-18}$.

Os sítios de tumor inicial foram bastante diversos, com as maiores prevalências no trato gastrointestinal e na mama, como encontrado por Souza et al. ${ }^{16}$. Dados nacionais do
Instituto Nacional de Câncer também apontam altos índices de câncer de mama ${ }^{3}$. Por sua vez, a porcentagem de metástases encontrada nesse estudo foi inferior ao observado por Silva et al. ${ }^{19}$, os quais analisaram 87 prontuários de pacientes em tratamento quimioterápico, constatando uma prevalência de metástase de 57,5\%. Quanto ao autorrelato de outras doenças crônicas, a maioria dos pacientes declarou ausência de comorbidades. Especificamente em relação aos indivíduos que declararam serem apenas hipertensos, outro estudo encontrou valor semelhante, de $22 \%{ }^{20}$.

Neste estudo, investigou-se tanto a alteração do comportamento alimentar quanto a prevalência de aversão alimentar apreendida, antes e depois do início do tratamento quimioterápico. Quanto à alteração do comportamento alimentar, observou-se que feiião, vegetais folhosos, legumes, leite e derivados, pães/bolos, sopas e refrigerantes/doces tiveram alteração significativa de consumo pelos pacientes. É válido ressaltar que essa mudança pode ser tanto de pacientes que consumiam um determinado alimento e deixaram de fazê-lo (por exemplo, café), como de indivíduos que não ingeriam determinado tipo de alimento e passaram a consumir (como as frutas). Marinho et al. ${ }^{9}$ também observaram alteração do comportamento alimentar em seu estudo, com uma diminuição da preferência por carnes, arroz, legumes, vegetais, doces e produtos de padaria no decorrer do tratamento quimioterápico, assim como um aumento do consumo de frutas, verduras e alimentos salgados. É válido ressaltar que essas alterações de comportamento podem refletir as 
questões sensoriais alteradas e os sintomas oriundos do tratamento ${ }^{20}$, mas também o conhecimento dos pacientes acerca da relação de determinados alimentos com a doença.

No presente estudo, os alimentos com maior aversão alimentar apreendida foram refrigerantes/doces, embutidos, massas, carnes e café. Outros estudos demonstraram que as carnes ${ }^{9,14,20}$, o cafée 910,14 e o feijão ${ }^{14}$ foram os alimentos com maior aversão alimentar apreendida durante 0 tratamento quimioterápico. As carnes e o café, embora não tenham sido os principais alimentos com aversão alimentar apreendida neste estudo, também apresentaram valores importantes e foram referidos pelas mesmas pessoas (dados não apresentados).

A aversão à carne pode estar relacionada ao limiar de amargura diminuído. Aminoácidos, peptídeos e purinas na forma pura têm um gosto amargo, sugerindo-se que, para pessoas saudáveis, a concentração destas substâncias na carne pode estar abaixo do limiar de reconhecimento amargo, enquanto num doente os mesmos compostos podem provocar uma sensação desagradável, resultando em aversão ao alimento. A redução do limiar de sabor amargo pode também estar relacionada com a aversão ao café, uma vez que a cafeína é considerada uma substância amarga? A carne é uma fonte importante de proteínas de alto valor biológico, minerais e vitaminas, e sua exclusão pode contribuir para perda de massa magra e agravos do estado geral de saúde do paciente oncológico ${ }^{21}$.

Alterações do paladar e do olfato estão entre os efeitos colaterais mais sofridos do tratamento quimioterápico, impactando seriamente na vida cotidiana dos pacientes oncológicos. Estudos demonstram que as alterações do paladar e do olfato durante a quimioterapia têm um impacto na vida do paciente em termos de funções domésticas (por exemplo, parceiros que assumem compras de mercearia e do preparo das refeições) e interações sociais (por exemplo, não comer fora ou convidar amigos para o jantar). Além disso, as alterações quimiossensoriais podem resultar em uma diminuição do prazer na alimentação ${ }^{22,23}$.

Neste estudo, a maioria dos pacientes informou não ter tido redução da ingestão alimentar, porém houve parcela importante de relatos de redução do sabor do alimento, xerostomia, enjoo oriundo do aroma dos alimentos e falta de apetite. Outros estudos também encontraram prevalência importante de sintomas que podem alterar a ingestão alimentar. Ponticelli et al. ${ }^{24}$ observaram que disgeusia foi desenvolvida após e durante a quimioterapia por $64 \%$ $(n=185)$ dos pacientes. Zabernigg et al. ${ }^{25}$ realizaram uma pesquisa com pacientes com câncer de pâncreas, colorretal ou de pulmão, concluindo que $69,9 \%(n=137)$ dos pacientes relataram alterações gustativas pelo menos uma vez. Já em um estudo feito com um grupo de pacientes, um mês após o último ciclo de quimioterapia, $65 \%$ dos sujeitos relataram piora no paladar, enquanto apenas 19\% referiram alterações negativas referentes ao olfato 22 .

Como uma forma de enfrentar a disgeusia, recorrente no tratamento quimioterápico, muitas estratégias foram adotadas pelos pacientes para amenizar este efeito ${ }^{24}$ : comer refeições pequenas e frequentes $(41 \%)$, beber mais água (33\%), alterar hábitos de tempero (33\%) e comer alimentos frios (19\%). Alguns pacientes $(25,7 \%)$ utilizaram uma combinação dessas estratégias. Essa alteração na função do gosto, decorrentes do tratamento, são cíclicas e transitórias, esperando-se que, após o término da quimioterapia, essas funções sejam regularizadas ${ }^{26}$. Assim, as intervenções destinadas a ajudar os pacientes a gerir autonomamente este efeito secundário são cruciais. Atentar a uma avaliação de risco adequada de disgeusia no início da quimioterapia pode prevenir o declínio nutricional e pode ajudar a manter uma ingestão de calorias adequada ${ }^{24}$.

Destaca-se como limitação deste estudo a falta de informação sobre tabagismo, o qual pode afetar a percepção de paladar e olfato, intensificando-se com a quimioterapia. A não averiguação do estado do paladar antes do tratamento, a alteração acometida por cada tipo de quimioterápico e a consideração de um possível viés de memória dos pacientes, em caso de pacientes que estão no tratamento há anos, também podem ser considerados como fatores limitadores. Salienta-se, ainda, a grande necessidade da contínua pesquisa sobre as aversões alimentares apreendidas e alterações de paladar e olfato, buscando entender suas causas, duração, para poder propor estratégias que melhorem a ingestão alimentar, o estado nutricional e qualidade de vida dos pacientes oncológicos em tratamento quimioterápico.

\section{CONCLUSÃO}

Neste estudo, pôde-se observar que houve uma importante alteração do comportamento alimentar durante o tratamento quimioterápico, afetando a ingestão de alimentos do cotidiano, o que pôde ser reforçado pela alta prevalência de alguns sintomas relacionados a alterações de paladar e olfato.

Portanto, destaca-se a importância da necessidade de uma contínua pesquisa sobre os efeitos da quimioterapia nos hábitos alimentares, tanto para compreender os efeitos desse tratamento, quanto para proporcionar ao paciente melhores orientações para contornar os efeitos do tratamento.

\section{REFERÊNCIAS}

1. Brasil. Organização Mundial da Saúde. Organização Pan-Americana da Saúde (OPAS). Folha informativa - Câncer. 2018. [cited 2019 Apr 15]. Available from: https://www.paho.org/ bra/index.php?option $=$ com_content $\&$ view $=$ article $\&$ id $=5588: \mathrm{f}$ olha-informativa-cancer\&Itemid $=1094$ 
2. Brasil. Ministério da Saúde, Instituto Nacional de Câncer. Consenso nacional de nutrição oncológica. $2^{\mathrm{a}}$ ed. rev. Rio de Janeiro: Ministério da Saúde, Instituto Nacional de Câncer; 2015.

3. Instituto Nacional de Câncer José Alencar Gomes da Silva. Estimate 2018: cancer incidence in Brazil. Rio de Janeiro: INCA; 2017. 128p.

4. Araújo LP, Sá NM, Atty ATM. Necessidades atuais de radioterapia no SUS e estimativas para o ano de 2030. Rev Bras Cancerol. 2016;62(1):35-42.

5. Instituto Nacional de Câncer José de Alencar Gomes da Silva (INCA). Tratamento do câncer. [cited 2019 Apr 15]. Available from: https://www.inca.gov.br/tratamento/quimioterapia

6. Tartari RF, Busnello FM, Nunes CHA. Perfil nutricional de pacientes em tratamento quimioterápico em um ambulatório especializado em quimioterapia. Rev Bras Cancerol. 2010;56(1):43-50

7. Calixto-Lima L, Martins de Andrade E, Gomes AP, Geller M, Siqueira-Batista R. Dietetic management in gastrointestinal complications from antimalignant chemotherapy. Nutr Hosp. 2012;27(1):65-75.

8. Monroy Cisneros K, Astiazarán García H, Esparza Romero J, Guevara Torres AG, Valencia Juillerat ME, Méndez Estrada $\mathrm{RO}$, et al. Antineoplastic treatment impact on nutritional status in patients with breast cancer. Nutr Hosp. 2014;30(4): 876-82.

9. Marinho EDC, Custódio IDD, Ferreira IB, Crispim CA, Paiva CE, Maia YCP. Relationship between food perceptions and health-related quality of life in a prospective study with breast cancer patients undergoing chemotherapy. Clinics. 2018;73:e411.

10. Verde SMML, São Pedro BMO, Mourão Netto M, Damasceno NRT. Aversão alimentar adquirida e qualidade de vida em mulheres com neoplasia mamária. Rev Nutr. 2009;22(6): 795-807.

11. Hernandez LS, Galve MIR. Influencia de las distorsiones sensoriales sobre el estado nutricional del paciente oncológico. Index Enferm. 2016;25(4):233-7.

12. Associação Brasileira de Empresas de Pesquisa. Critérios de classificação econômica. Brasil. 2018. [cited 2019 Apr 15]. Available from: http://www.abep.org/criterio-brasil

13. Gonzalez MC, Borges LR, Silveira DH, Assunção MCF, Orlandi SV. Validação da versão em português da avaliação subjetiva global produzida pelo paciente. Rev Bras Nutr Clin. 2010;25(2):102-8.

14. Ferreira NMLA, Scarpa A, Silva DA. Quimioterapia antineoplásica e nutrição: uma relação complexa. Rev Eletr Enf. 2008;10(4):1026-34.
15. Descritores em Ciências da Saúde: DeCS [Internet]. ed. 2017. São Paulo (SP): BIREME/OPAS/OMS. 2017. [cited 2020 Jun 25]. Available from: http://decs.bvsalud.org

16. Souza RS, Simão DAS, Lima EDRP. Perfil sociodemográfico e clínico de pacientes atendidos em um serviço ambulatorial de quimioterapia paliativa em Belo Horizonte. Rev Min Enferm. 2012;16(1):38-47.

17. Bastos BR, Pereira AKS, Castro CC, Carvalho MMC. Perfil sociodemográfico dos pacientes em cuidados paliativos em um hospital de referência em oncologia do estado do Pará, Brasil. Rev Pan-Amaz Saude. 2018;9(2):31-6.

18. Andrade FP, Muniz RM, Lange C, Schwartz E, Guanilo MEE. Economic and socio-demographic profile of cancer survivors according to degree of resilience. Text Context Nursing. 2013;22(2):476-84.

19. Silva MM, Silva JA, Esteves LO, Mesquita MGR, Stipp MAC, Duarte SCM. Perfil sociodemográfico e clínico de pessoas em tratamento quimioterápico: subsídios para o gerenciamento em enfermagem. Rev Eletr Enf. 2013;15(3):704-12.

20. Silva AM, Franco LP, Santos TSS, Passos XS, Costa BMF. Impacto das aversões alimentares no estado nutricional de pacientes oncológicos submetidos à quimioterapia. J Health Sci Inst. 2012;30(2):166-70.

21. Gangadharan A, Choi SE, Hassan A, Ayoub NM, Durante G, Balwani S, et al. Protein calorie malnutrition, nutritional intervention and personalized cancer care. Oncotarget. 2017;8(14):24009-30.

22. Vries YC, Boesveldt S, Kelfkens CS, Posthuma EE, van den Berg MMGA, Kruif JTCM, et al. Taste and smell perception and quality of life during and after systemic therapy for breast cancer. Breast Cancer Res Treat. 2018;170(1):27-34.

23. Vries YC, Helmich E, Karsten MD, Boesveldt S, Winkels RM, van Laarhoven HW. The impact of chemosensory and foodrelated changes in patients with advanced oesophagogastric cancer treated with capecitabine and oxaliplatin: a qualitative study. Support Care Cancer. 2016;24(7):3119-26.

24. Ponticelli E, Clari M, Frigerio S, De Clemente A, Bergese I, Scavino E, et al. Dysgeusia and health-related quality of life of cancer patients receiving chemotherapy: a cross-sectional study. Eur J Cancer Care (Engl). 2017;26(2). doi: 10.1111/ecc.12633.

25. Zabernigg A, Gamper EM, Giesinger JM, Rumpold G, Kemmler $\mathrm{G}$, Gattringer $\mathrm{K}$, et al. Taste alterations in cancer patients receiving chemotherapy: a neglected side effect. Oncologist. 2010;15(8):913-20

26. Boltong A, Aranda S, Keast R, Wynne R, Francis PA, Chirgwin J, et al. A prospective cohort study of the effects of adjuvant breast cancer chemotherapy on taste function, food liking, appetite and associated nutritional outcomes. PLoS One. 2014;31;9(7):e103512.

Local de realização do estudo: Universidade Federal de Pelotas (UFPel), no setor de Quimioterapia do Hospital Escola, Pelotas, RS, Brasil.

Conflito de interesse: Os autores declaram não haver. 